\title{
6-Gingerol: A Novel Therapeutic Approach towards Corona Virus
}

\author{
Ritesh Kumar and Kapil Hazarika \\ Department of Pharmacology Shree Guru Gobind Singh \\ Tricentary University, Gurugram, Haryana, India. \\ Corresponding author email: kapil_fmhs@sgtuniversity.org
}

\section{ABSTRACT}

In December 2019, COVID-19 emerged as a pandemic which was identified as Severe Acute Respiratory Syndrome Corona Virus (SARS COV2). World Health Organisation (WHO) confirmed it as Global health crisiswhich affected lifestyle and economy of many countries. The evolution of pathogen virus causes changes in amino acid sequences which has posed difficultiesin developing a vaccine against this disease. The present study is based on evaluation of natural therapeutics for treatment of Covid-19. Ginger which has main constituent as 6-gingerol,has been used to treat many respiratory disorders. In this study, In-silico studies were performed using autodock4 to study the interaction between 6-gingerol and S-Protein or Spike protein of SARS CoV2. The results indicate that Phytocompound 6-gingerol exhibited good binding energies ranging from $-0.99 \mathrm{kcal} / \mathrm{mol}$ to $-3.25 \mathrm{kcal} / \mathrm{mol}$ with the S-protein . This research suggests that 6-gingerol can be a potent anti-viral phytocompound to develop a vaccine against corona virus. The study provides a natural copound as Covid-19 treatment, which could be further studied by in-vitro and clinical research methods.

KEY WORDS: ANGIOTENSIN CONVERTING ENZYME 2(ACE2), COVID-19, SPIKE PROTEIN, SARS COV2, ,ZINGIBEROFFICINALE.

\section{INTRODUCTION}

Corona virus or SARS CoV2 has caused a pulmonary disease all over the world, but it all started in December 2019 in the city of Wuhan, China. On 11 March 2020, World health Organisation (WHO) announced that this disease is pandemic. On May 16 2020, there were more than 4.59 million cases all over the world and till now there are 36 million cases globally (Figure 1) as per the reports given by the WHO(Worldometer et. al. (2020)). SARS CoV2 got its name from the Severe Acute Respiratory Syndrome Corona Virus (SARS CoV) because of having about $82 \%$ of similar genomic RNA. Till now there are seven types of strains of Corona Virus are known in which four strains; 229E, NL63, OC43 and HKU1 are found to cause mild symptoms like cold and cough where as the other three

Biosc Biotech Res Comm P-ISSN: 0974-6455 E-ISSN: 2321-4007

\section{crossef}

Identifiers and Pagination

Year: 2021 Vol: 14 No (7) Special Issue

Pages: 273-277

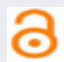
creative Article Information Received: $9^{\text {th }}$ May 2021

This is an open access article under Creative

Commons License Attribn 4.0 Intl (CC-BY).

DOI: $h t t p: / / d x . d o i . o r g / 10.21786 / b b r c / 14.7 .60$ corona virus; MERS CoV, SARS CoV and SARS CoV2 are likely to cause the severe symptoms which may lead to death in severe cases.

Figure 1: Total cases of COVID-19 reported Globally (Worldometer et. al. (2020)

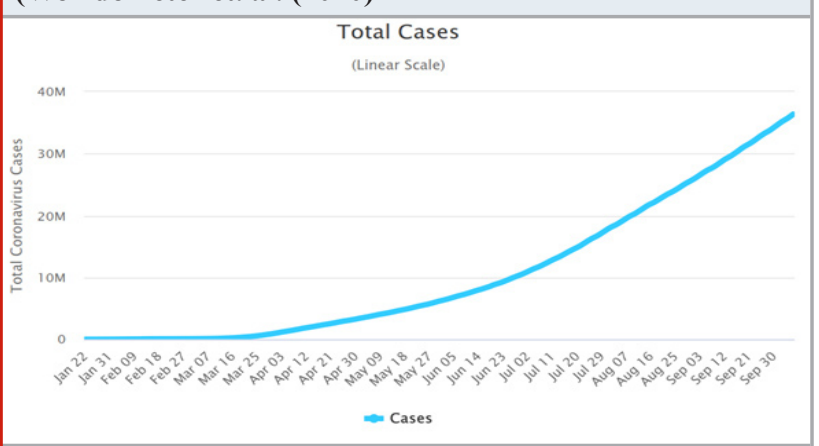

Structure of Corona Virus: All the corona viruses including SARS CoV2 have lipid envelope, positive sense RNA and with the help of host derived membrane it wraps the viral genome and contains it. SARS CoV2 are round in shape and have proteins called spikes or s-proteins sticking out on their surfaces (Figure. 2). These s-proteins are responsible for the fusion of viral and cell membrane by fastening into

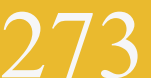


the human cell. After the fusion, the viral genes enters the human cells and by hijacking the replication machinery they start producing more viruses inside the human body which further attacks the other host cells. Recent researches showed that Angiotensin-converting enzyme-1 or ACE2 on human cell surface is acting as a receptor to the S-protein or spike protein. It is interesting to know that before the SARS CoV2 S-Protein can link with the ACE2 of human cell, it under goes the structural changes to represent its receptor binding domain or the piecethat will interact with the human cell ACE2(S. Kumar et. al. (2020)).

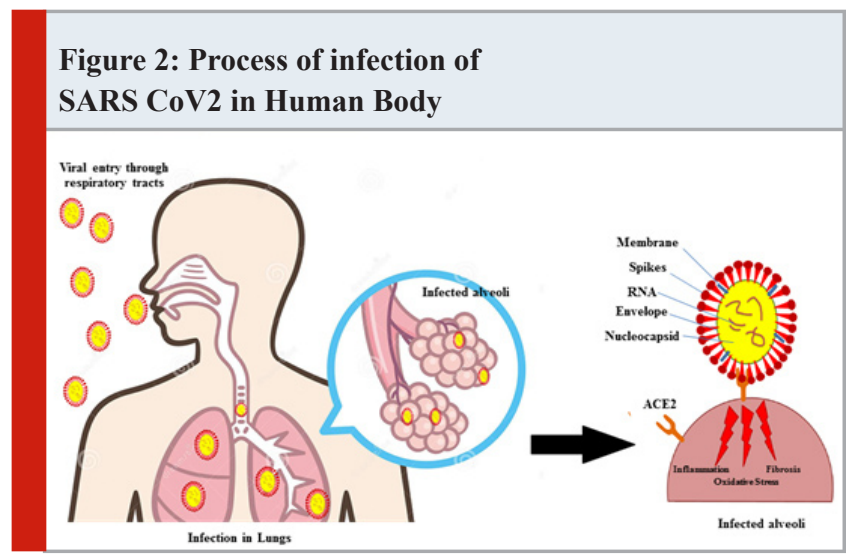

Like many other viral proteins, The S-Protein needs to be shielded from the host immune system, so it hasa thick layer of glycans on its outer surface. These glycans not only provide shield to the S-protein but these are also present at the specific sites to stop the host immune system more efficiently. This SARS CoV2 S-Protein can be targeted for the development of the vaccine againstthe SARS CoV2.

Figure 3: Types of 4-hydroxy-3-methoxyphenyl)methyl 7-methyloctanoatepresent in Ginger.

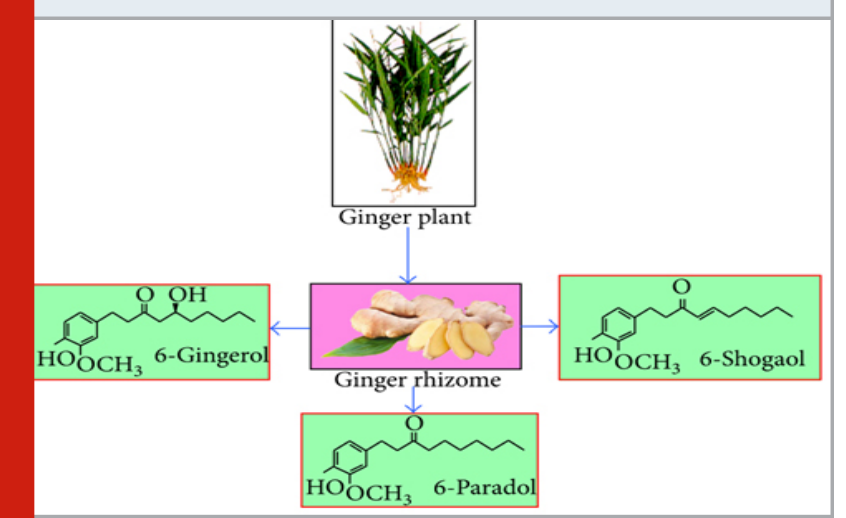

Natural Products: Natural products yielding medicinal properties are always have been in the great interest as they play an essential role in preventing and curing many human life threatening diseases. India is a country where many spices and trees are used to treat many diseases. Many active ingredients having a high pharmaceutical potentialhave beenfound and extracted for developing novel medicines. These pharmaceutical gradients depicts that they are of very much importance in the matter of physiological and pharmacological properties. It is a challenge to find out anti-Corona Virus compounds out of so many active products that can be possibly be used to treat Corona Virus. In this study, we have selected medicinal plant Zingiberofficinale(Ginger).

Ginger is a traditional medicinal plant with many proved medicinal applications. It is also used in many biological and therapeutic strategiesto treat diseases. There are many chemical constituents of Ginger, but the majority of the health benefits come with its phenolic compounds. 6-Gingerol is major chemical constituent of ZingiberOfficinale and it have been proved that it cures various types of respiratory disorder There are 3 forms of 4-hydroxy-3-methoxyphenyl) methyl 7-methyloctanoate (Figure. 3) found namely: 6-gingerol, 6-shogaol and 6-paradol. In this research 6-gingerol is selected as it has proved its potential in treating many diseases.(B.J.Oso et. al. (2020).

Literature Review: Rajagopal, K., et al. in an article "Activity of phytochemical constituents of black pepper, ginger, and garlic against Corona Virus (COVID-19): An in silico approach" stated the in-silico studies on 8-Gingerol and 10-Gingerol on the COVID-19 and found that both the phenolic compounds were significantly active against the COVID-19, but they haven't stated the effects of 6-gingerol, here this research is focusing on the effect of 6-gingerol on the S-protein of SARS CoV2(P. Sudhir et. al. (2018). Another study conducted by Ghosh et al., discussed the roles of green tea polyphenols by studying the binding affinity of eight polyphenols of natural compounds found in green tea with the Mpro of SARS CoV2. This research shows that there are chances that other phytocompunds can have higher binding affinity towards other proteins of SARS CoV2 which can help us to further develop a better vaccine.

Kumar, S., et.al. in an article "Identification of phytochemicals as potential therapeutic agents that binds to Nsp5 protein target of Corona Virus (SARS-CoV-2) that are capable of inhibiting virus replication" showed the binding potential of 50 photochemicals with the SARS CoV2 Nsp5 and found that hydrogen bonds, docking results and DeltaG value using insilico studies(S. Kumar et. al. (2020). Oso, B.J., et.al.Presented in a journal"Pharmacoinformatics and hypothetical studies on allicin, curcumin, and gingerol as potential candidates against COVID-19-associated proteases'. In this journal Oso, B.J., et.al. Stated the comparison between different phyto-compounds and Oso, B.J., et.al. found that curcumin have the highest binding affinity as compared to the others, (B.J.Oso et. al. (2020).

Rathinavel, T., et.al. Have performed a research on topic "Phytochemical 6-Gingerol- A promising Drug of choice for COVID-19". Rathinavel, T., et.al. performed in-silico studies with 6-gingerol on 12 SARS CoV2 proteins but they didn't performed the studies on the S-Protein, here in this research, in-silico studies are done on the interaction between 6-gingerol and S-Protein. Different energies are recorded and based on those energies it was concluded that whether 6-gingerol can be a phytocompound to prevent SARA CoV2(T. Rathinavel et. al. (2020). In previously already done researches, there is no mention of the $\mathrm{H}$-bond energy, bind affinity, intermolecular energy electrostatic 
energy and total internal energy of 6-gingerol and SARS CoV2 interaction. Here in this research, various in-silico studies are performed on which different energies are recorded. On the basis of these energies it will be concluded whether 6-gingerol can be a good phytocompound to treat the SARS CoV2 or not. This research discusses the insilico studies done on 6-Gingerol and the Spike protein of SARS CoV2. These studies will further help the reader to understand why 6-Gingerol can be used in the development of a better drug that can be used to cure or prevent human beings from SARS CoV2.

Research Questions: Can 6-gingerol show interaction and docking with $\mathrm{S}$ protein of Corona virus?

\section{METHODOLOGY}

Design: As Shown in Figure 4, 3D structure of 6-gingerol was downloaded from PubChem and 3D tertiary structure of SARS CoV2 S-Protein was downloaded from RCSB PDB. After downloading these files are formatted to a same file format as .pdb and then these two files are used for docking. Within the AutoDock software there are series of steps where .pdbqt, .glg, .dlg files are formed. .dlg file type is the docked file which can be visualised only in selected tools like AutoDock, Discovery Studio and PyMol. In this research .dlg file was visualised in Autodock and docked models with different estimated free energy of binding was recorded.

Figure 4: Flow chart representing the experimental protocol of the study.

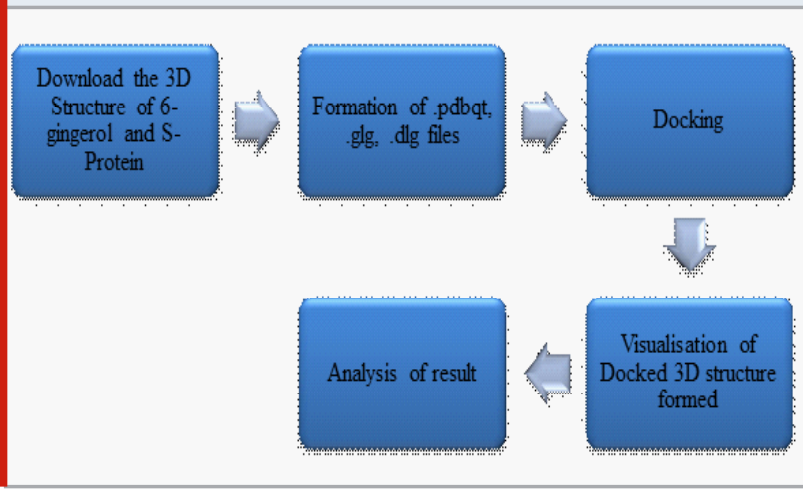

Sample: 3D structures help us to better understand the properties of molecules. Figure 5 shows the 3D structure of 6-gingerol downloaded from PubChem (National Library of Medicine et. al. (2020), the grey balls depicts the carbon atoms, red balls are depicting the oxygen atoms and white balls are for hydrogen atom. Figure 6 is the Tertiary Structure of Spike protein downloaded from RCSB PDB where the spiral ribbons are the alpha helix and the straight ribbons shows the beta plated sheets.

Instrument: The following databases and softwares have been used for conducting in-silico studies of 6-Gingerol and S protein:

Public Chemical (PubChem): SDF formal file was downloaded for 3D structure of 6-Gingerol from PubChem (Figure5). The open data base for chemistry compounds
Figure 5: Ball and stick model of 6-Gingerol obtained fromPubChem(National Library of Medicine et. al. (2020)

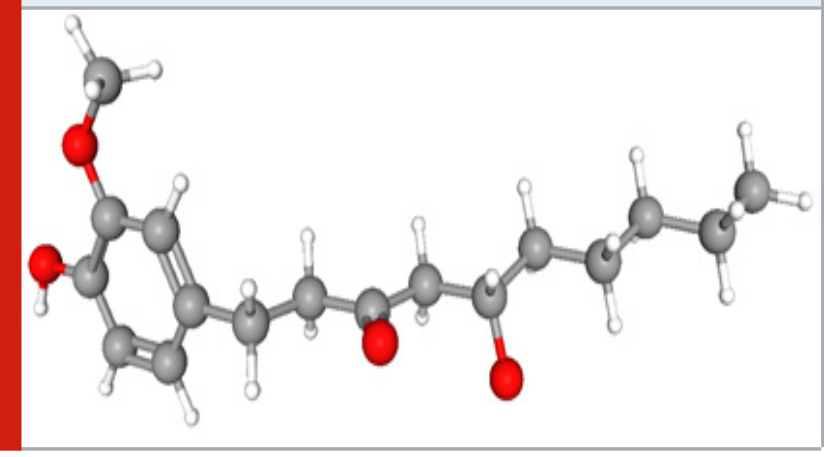

Figure 6: Tertiary structure based 3D model of S-Protein

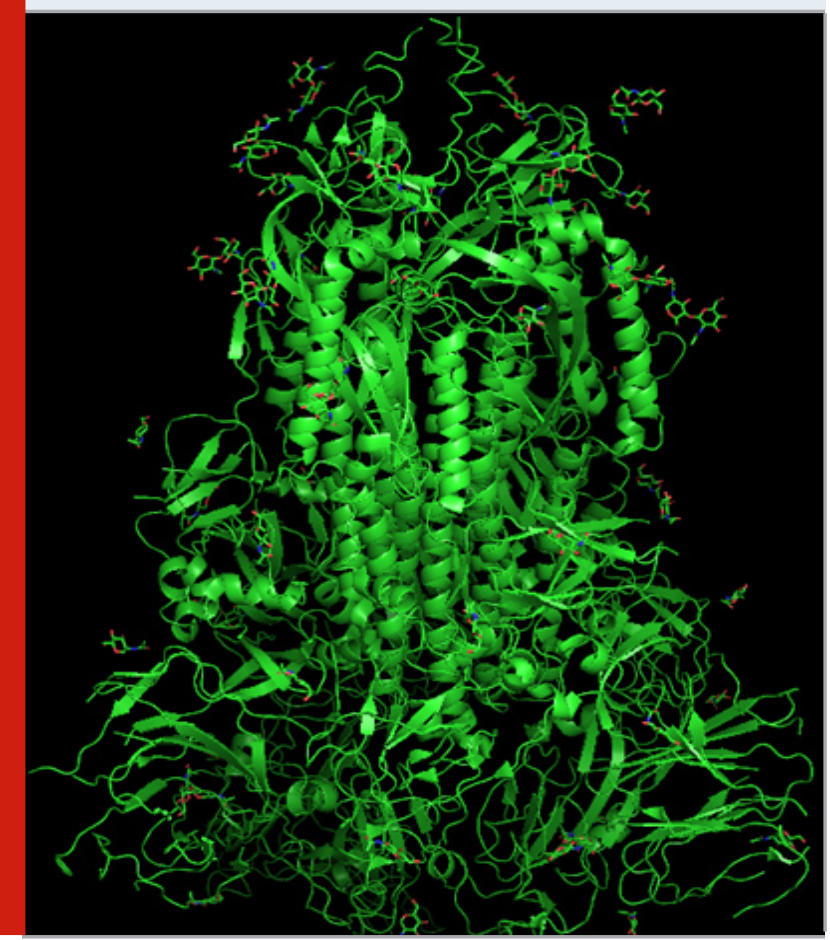

was used to search structures by chemical name, formula, structure and other keywords. Research Collaboratory for Structural Bioinformatics Protein Data Bank (RCSB PDB):

Downloading the PDB format of S-protein from RCSB (Figure6). RCSB PDB is an open accessible protein data bank that can be accessed online. It is a downloadable data archive which provides 3D conformation of all biological substances and medicines.

Open Babel: After downloading the Ligand and protein, downloaded files were converted to PDB format with the help of Open Babel. Open Babelis a open easily accessible tool used for chemical data to convert to different file formats, store or analyse the data.

AutoDock: AutoDock is used to dock the compound on 
the viral protein and after docking Binding energies were calculatedAutoDockis an open and easily accessible tool that is used to calculate how one small molecule will bind or how will it interact with the receptor of a known structure in 3D. Apart from docking it also calculates the binding affinity that helps in finding a better binding molecule to a receptor.

Data Collection: After Docking 10 Docked models of 6-gingerol on SARS CoV2 Spike protein was obtained at 10 different locations on protein as can be observed from figure 7 and 8 . These 10 docked models depicts that 6-gingerol is binding with the spike protein of SARS Cov2. Every docked model have a different estimated free energy of binding. Out of these 10 docked models, model 2 was selected because of having the least estimated free energy of binding.

\section{Figure 7: 10 Docked models obtained by Autodock}

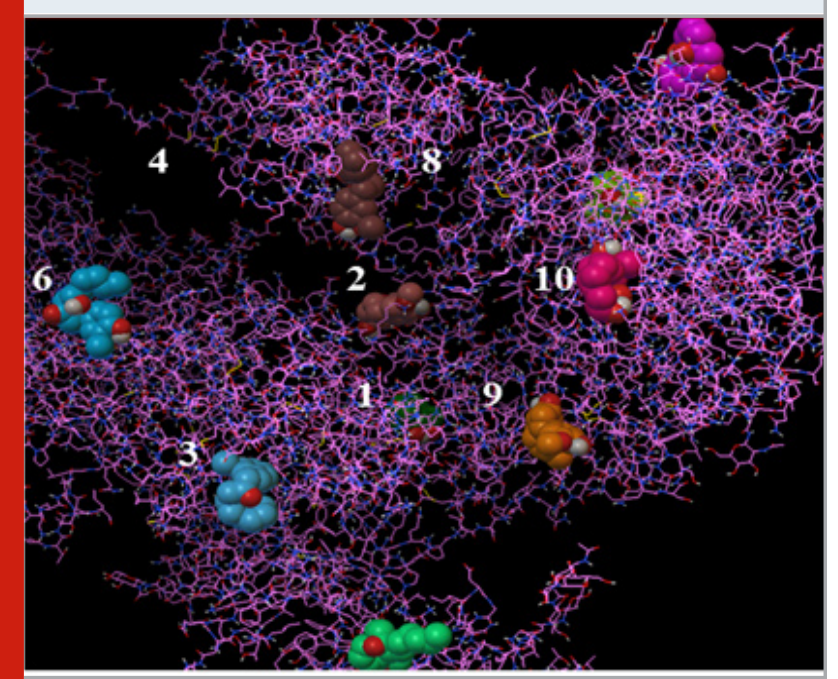

Data Analysis: All the 10 Docking models was analysed using AutoDock. Various parameters like Free energy of binding, inhibition constant, intermolecular energy, vdW + Hbond + desolv Energy and total internal energy were compared and the free energy of binding was ranging from $-0.99 \mathrm{kcal} / \mathrm{mol}$ to $-3.25 \mathrm{kcal} / \mathrm{mol}$ as shown in Table 1 .It was calculated that Model no. 2,8 and 10 were the three models that were having the least Free energy of binding.

\section{RESULTS AND DISCUSSION}

After performing the in-silico studies on the compound and the Spike protein of SARS CoV2, it was found that there are 10 docking sites where the 6-gingerol is docked as shown in figure 7. With the help of Autodock4 it was observed that the different energies and the Estimated Free energy of binding were responsible for docking of 6-Gingerol. Estimated free energy of binding is calculated on the bases of negativity, the more the negative value means better binding between the atoms of phyto-compound and the viral protein. On comparing the estimated free energy of binding of all 10 Models (Figure 7)shown in Table 1 it was found that the Docked Model Number 2 (Figure8)has better energy as compared to other Docked Models.

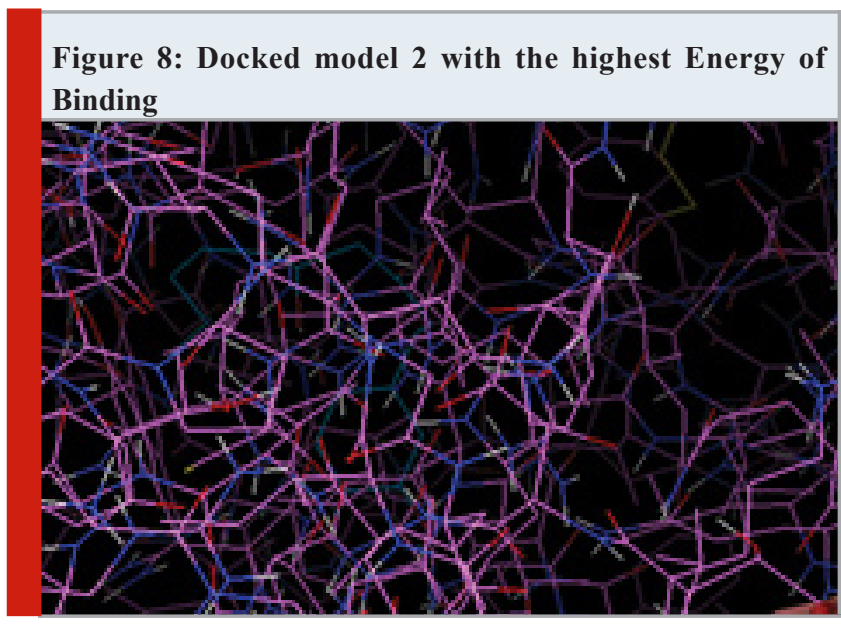

CONCLUSION

Aim of the current study was to screen the pre-existed antiviral phyto-compounds and find out the potential inhibitor of SARS CoV2 S-Protein. The benefit of computational studies is that they reduce the risk of drug failing, reduce the cost of clinical trials and reduce the time taken to develop the drug. Since, developing a potent drug against any disease takes a lot of time which is not affordable at this time of COVID-19, as it is spreading at a very rapid rate. The proposed phyto-compound showeda efficient binding energy and 6-Gingerol showed a free energy of binding of $-3.25 \mathrm{kcal} / \mathrm{mol}$ and it is suggested that it could be the efficacious therapeutic potential phytocompound against COVID-19. However, further in-vitro tests are required to evaluate the potential of 6-gingerol as inhibitor for COVID-19.

\section{REFERENCES}

Ezhilarasi, G.D., Latchoumi, T.P. and Balamurugan, K., 2020. UIP - A Smart Web Application to Manage Network Environments, Advances in Intelligent systems and computing book series.

Ghosh, R., Chakraborty, A., Biswas, A. and Chowdhuri, S., 2020. Evaluation of green tea polyphenols as novel corona virus (SARS CoV-2) main protease (Mpro) inhibitors-an in silico docking and molecular dynamics simulation study. Journal of Biomolecular Structure and Dynamics, pp.1-13.

Kumar, S., Kashyap, P., Chowdhury, S., Kumar, S., Panwar, A. and Kumar, A., 2021. Identification of phytochemicals as potential therapeutic agents that binds to Nsp 15 protein target of coronavirus (SARS-CoV-2) that are capable of inhibiting virus replication. Phytomedicine, 85, p. 153317.

Mishra, P., Jimmy, L., Ogunmola, G.A., Phu, T.V., Jayanthiladevi, A. and Latchoumi, T.P., 2020, December. Hydroponics cultivation using real time iot measurement system. In Journal of Physics: Conference Series (Vol. 1712, No. 1, p. 012040). IOP Publishing.

Oso, B.J., Adeoye, A.O. and Olaoye, I.F., 2020. 
Pharmacoinformatics and hypothetical studies on allicin, curcumin, and gingerol as potential candidates against COVID-19-associated proteases. Journal of Biomolecular Structure and Dynamics, pp.1-12.

Prabhu, S. and D'Cunha, D., 2018. Comparison of body image perception and the actual BMI and correlation with self-esteem and mental health: A cross-sectional study among adolescents. Int. J. Health Allied Sci, 7, pp.145149.

Prasath, S. (2020). Probabilistic Mceliece Public-Key Cryptography Based Identity Authentication For Secured Communication In Vanet. Solid State Technology, 63(6), 10167-10182.
Prasath, S. Validating Data Integrity in Steganographed Images using Embedded Checksum Technique. International Journal of Computer Applications, 975, 8887.

Rathinavel, T., Palanisamy, M., Palanisamy, S., Subramanian, A. and Thangaswamy, S., 2020. Phytochemical 6-Gingerol-A promising Drug of choice for COVID-19. Int J Adv Sci Eng, 6(4), pp.1482-9.

Venkata Pavan, M., Karnan, B. and Latchoumi, T.P., 2021. PLA-Cu reinforced composite filament: Preparation and flexural property printed at different machining conditions. Advanced Composite Materials, https://doi. org/10.1080/09243046.2021, 1918608. 